\title{
BMJ Open Healthcare professionals' experience of using in situ simulation training in preparation for the COVID-19 pandemic: a qualitative focus group study from a Danish hospital
}

\author{
Joachim Juelsgaard (D) , ${ }^{1}$ Bo Løfgren, ${ }^{2,3}$ Neel Toxvig, ${ }^{4}$ Gitte Valsted Eriksen, ${ }^{5}$ \\ Lotte Ebdrup, ${ }^{6}$ Rune Dall Jensen ${ }^{4}$
}

To cite: Juelsgaard J, Løfgren B, Toxvig N, et al. Healthcare professionals' experience of using in situ simulation training in preparation for the COVID-19 pandemic: a qualitative focus group study from a Danish hospital. BMJ Open 2022;12:e056599. doi:10.1136/ bmjopen-2021-056599

- Prepublication history and additional supplemental material for this paper are available online. To view these files, please visit the journal online (http://dx.doi.org/10.1136/ bmjopen-2021-056599).

Received 17 September 2021 Accepted 20 December 2021

Check for updates

(C) Author(s) (or their employer(s)) 2022. Re-use permitted under CC BY-NC. No commercial re-use. See rights and permissions. Published by BMJ.

For numbered affiliations see end of article.

Correspondence to Dr Joachim Juelsgaard; juelsgaardjunior@hotmail.com

\section{ABSTRACT}

Objectives The COVID-19 pandemic forced hospital organisation and healthcare professionals to prepare for large quantities of patients in isolation rooms. In situ simulation may seem promising in order to manage the organisational changes that the pandemic require. This study aims to investigate in situ simulations influence on healthcare professional's self-perceived preparedness to face the pandemic.

Design A qualitative focus group study.

Setting We conducted full scale in situ simulations over a 3-week period in April 2020, including 277 healthcare professionals, at a Danish University Hospital. Subsequently, six semistructured focus group interviews, including 22 participants from the simulations, were conducted in May 2020.

Participants 22 healthcare professionals participated in the focus group interviews.

Methods The simulations consisted of a briefing, two scenarios focusing on acute respiratory insufficiency and correct use of personal protective equipment (PPE), and a debriefing. We conducted six focus group interviews using comparable semistructured interview guides focusing on the organisational restructuring of the departments and outcomes of the needs-driven simulation-based programme. We used thematic analysis to identify main themes.

Results The informants perceived that the simulations resulted in positive experiences for the healthcare professionals and perceived the organisational changes as effective. They highlighted that simulation enhanced teamwork, demystified the COVID-19 disease, and improved skills, in correct use of PPE and acute treatment of COVID-19 patients. Data revealed that a predefined simulation task force including both experienced simulators and medical experts for facilitation of in situ simulation would be beneficial.

Conclusion In situ simulation may be useful to enhance learning on organisation and individual level during a pandemic. This educational activity could serve an important role in facilitating hospital preparation and education of large numbers of healthcare professionals during a healthcare crisis. Introduction of a simulation
Strengths and limitations of this study

- The study presents insights based on healthcare professionals' experiences with participating in COVID-19 in situ simulations in a university hospital.

- A focus group interview intervention of involved healthcare professionals was performed in close timely relation to the simulations.

- In situ simulation facilitated learning both at the individual and organisation levels.

- Due to the pandemic a limited number of informants are included in this study.

task force is suggested to handle coordination and rapid enrolment across the hospital.

\section{INTRODUCTION}

SARS-CoV-2 has been spreading worldwide since its occurrence by the end of 2019, causing the COVID-19 pandemic. WHO estimated a global mortality at $3.4 \%$ in the initial phase of the pandemic. ${ }^{1}$ These numbers pressured the existing workforce, and called for an increase in numbers of healthcare professionals, who could act as front-line staff during the pandemic. ${ }^{2}$ Vagni et al found that healthcare professionals involved in the treatment of COVID-19 were exposed to a large degree of stress, especially if they lack adequate knowledge about the disease. ${ }^{3}$

Training and correct use of personal protection equipment (PPE) in the care of all patients with respiratory symptoms was essential due to contamination risks. ${ }^{45}$ Hence, the immense pressure on the healthcare system called for immediate development of justin-time preparedness strategies in order to meet the challenges of new healthcare professionals not familiar with the disease, risk of 
Phase 1: 84 beds - next phase activated at 50\% of capacity

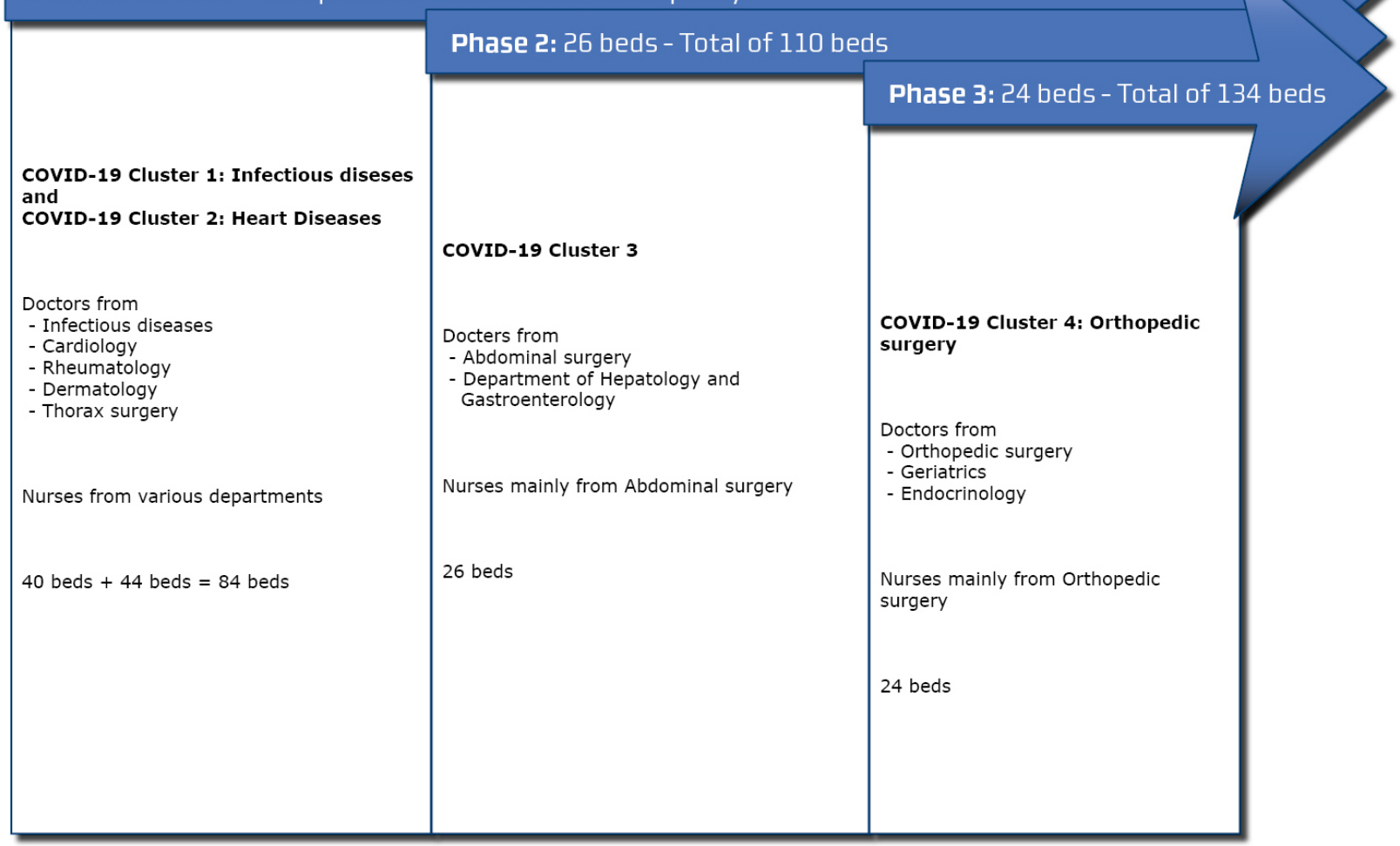

Figure 1 COVID-19 clusters, Aarhus University Hospital. March 2020-isolation rooms.

contamination, and risk of mental health issues among the professionals. ${ }^{6}$

In situ simulation has a potential to help managing the global COVID-19 crisis and in potentially similar future pandemics. ${ }^{2-9}$ It is, however, not known how the use of in situ simulations affected the healthcare professionals, due to the rare occurrence of pandemics. Thus, this study aims to investigate how healthcare professionals, educational experts and leaders at department levels at a University Hospital perceived their involvement in an in situ simulation programme.

\section{Reorganisation of the hospital and design of COVID-19 clusters}

Aarhus University Hospital, which contains above 1200 bedsides, established a COVID-19 clinic focused on COVID-19 testing, identification and triaging.

In addition, the capacity at the Department of Intensive Care were increased and four COVID-19 clusters were established, with a total capacity of 134 isolation rooms. The term COVID-19 clusters refers to the fact that several medical specialties worked together at one isolation ward. See figure 1 for details.

A stepwise approach was used in order to convert four in-patient departments to COVID-19 patient treatment clusters. The clusters should admit COVID-19 patients sequentially when $50 \%$ of the capacity of the previous cluster were in use, however only COVID-19 cluster 1 and 2 were activated during the study period. The allocated healthcare professionals had their daily work in a variety of clinical departments and faced unfamiliar working routines, with respect to colleagues, teams and working locations within the COVID-19 clusters.

\section{Educational activities in the COVID-19 clusters}

In order to prepare personnel, a steering committee in each cluster was established. As a part of the steering committee, educational teams were established in order to prepare the healthcare professionals to face the pandemic. Coauthor LE was part of the educational team in cluster 1 and coauthor BL was part of the educational team in cluster 2. As part of the educational activities an in situ simulation programme was developed. In situ simulation was used as a method due to an educational focus on individual and organisational learning. ${ }^{2}$ The programme had an agile structure making it adjustable in regards to the continuous development of new guidelines about triaging, resuscitation, and treatment of COVID-19 patients.

Experiences and insights from the simulation was shared with the hospital administration on a daily basis, in order to ensure that gained knowledge and points of attention could benefit the entire hospital. Hence, the understanding of COVID-19 disease and how to improve the organisation of isolation rooms 


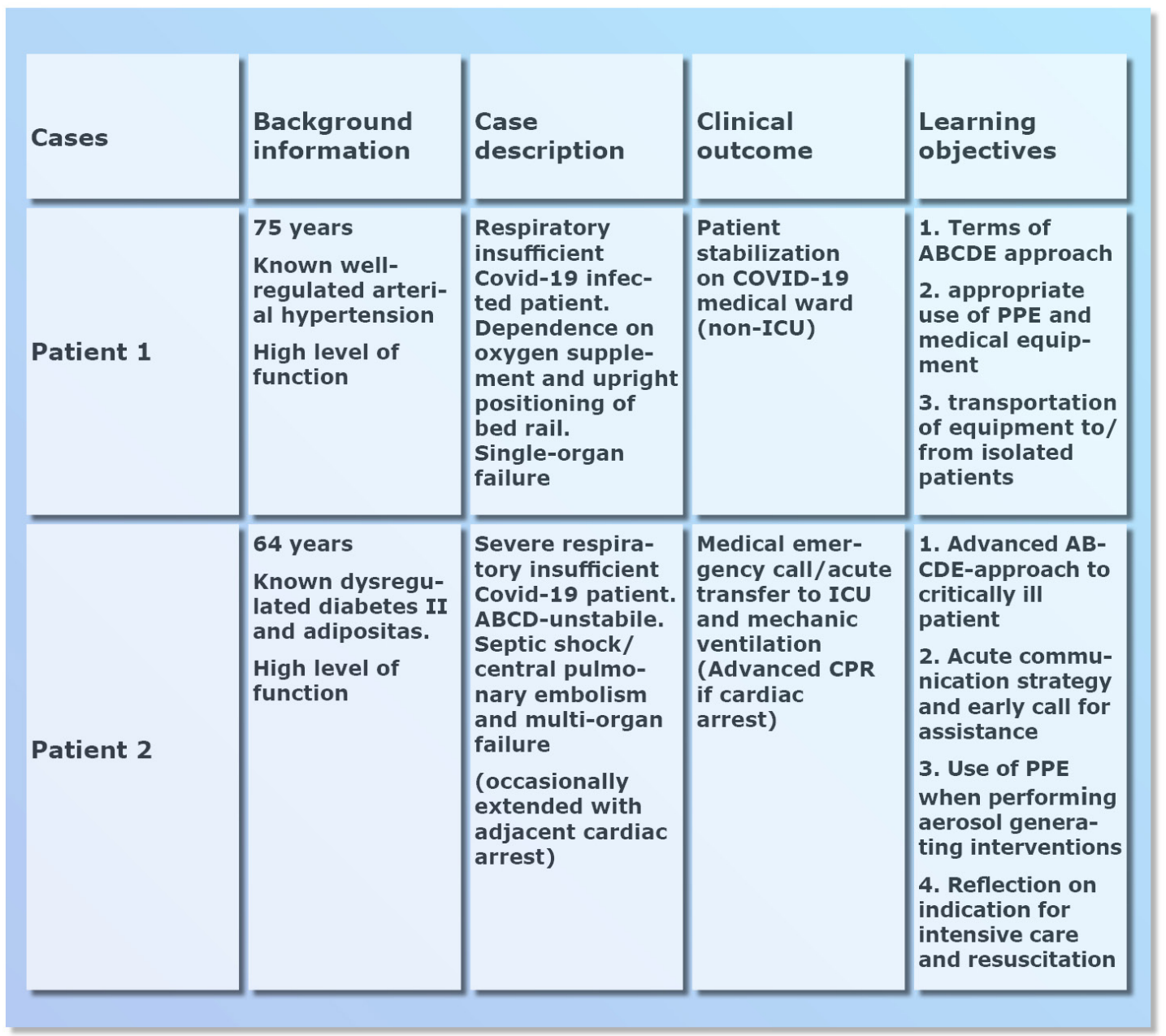

Figure 2 Description of simulated cases. PPE, personal protective equipment. ICU, intensive care unit. ABCDE, Airway, Breathing, Circulation, Disability and Exposure. CPR, Cardiopulmonary resuscitation.

and clinical teams were shared based on insights from the simulations. In this way, there was a close relation between the different levels of management at the hospital.

\section{METHODS}

We conducted six qualitative focus group interviews including a total of 22 healthcare professionals. The focus group method and a narrative research approach can be used to investigate meanings and beliefs that influence the informants' attitudes toward in situ simulation as an educational tool. ${ }^{10}$ The informants were participants from a simulation based educational initiative focusing handling and treatment of COVID-19 patients. These simulations took place at Aarhus University Hospital and included a total of 277 healthcare professionals (doctors, nurses, and physiotherapists) and were conducted during a 3-week period in April 2020. The team based in situ simulations lasted between 60 and $90 \mathrm{~min}$ and consisted of two scenarios focusing on acute respiratory insufficiency, correct use of personal protective equipment (PPE), team communication and transportation of unstable patients, please see figure 2. The participants all simulated both scenarios. All simulations were facilitated by a medical expert and an educated simulation instructor, both present during briefing, conduction and debriefing of the scenarios. The participants were organised in groups of 4-6 individuals in a team composition that corresponded to the clinical teams in the newly established COVID-19 clusters (1-2 doctors, 2-4 nurses and 1-2 other healthcare professionals). A manikin deputised as patient and all equipment from a basic medical ward was available. Measured vitals was presented on a monitor and operated by the facilitators during the scenarios.

The simulations did not include formal assessments of the learning due to the time-sensitive nature of the training. Instead, the simulations included all healthcare professions that treated COVID-19 patients and all participants were asked to actively participate in the simulation and in giving and receiving feedback. The scenarios in the simulation sessions required collaborative and active learning. The simulations were conducted as rapid cycle deliberate practice, consisting of average $20 \mathrm{~min}$ briefing, 20 min scenario and 20 min debriefings. ${ }^{11}$ Rapid cycle deliberate practice was selected as Hunt et al showed that this approach is associated with improvement in 
performance of key measures and progressive acquisition of trained skills during simulation. ${ }^{11}$ Sessions were debriefed using teamGAINS ${ }^{12}$ as technical and non-technical leaning objectives were essential for the outcome.

\section{Data collection}

The qualitative focus group interviews lasted between $35 \mathrm{~min}$ and $63 \mathrm{~min}$ per interview using comparable semistructured interview guides. ${ }^{13}$ The interview (see full interview topic guide in online supplemental material) guides comprised thematically structured open-ended questions with respect to themes as uncertainty, fear of contamination and lack of preparedness among healthcare professionals during the pandemic, which is known from the educational literature. ${ }^{2} 710$ Furthermore, informants were asked to reflect on how the COVID-19 pandemic affected their daily work routines. The themes of the interviews can be seen in the interview topic guide. The semistructured interview guide allowed the interviewer to probe for additional insight and to dig deeper into the pros and cons of the simulations. The interviewers were trained qualitative researchers who also facilitated the simulations. However, each cluster had its own facilitators thus, the interviewers did not interview participants they had trained. This was done in order to decrease the power relation between interviewer and interviewee. The informants in this study were included based on their experience with being either participants in simulations, facilitators of the simulations, members of the educational committee or consultants responsible for the included departments. We included doctors, nurses and other healthcare professionals in order to embrace the voice of all professions. More nurses than doctors were included in order to reflect the composition of the clinical teams from the simulations. We planned for 4-7 informants in each group. However, in the focus group consisting of the consultants responsible for the clusters only three informants participated. Each focus group had a strategic composition of informants in order to decrease the power differential among the informants according to the methodological recommendations from Stalmeijer et al. ${ }^{14}$ All informants were recruited as volunteers.

We applied a qualitative methodology that relates to the social constructionist and narrative understanding of storytelling as being integral to the analysis of healthcare professionals' perspectives and personal experiences when dealing with the pandemic. ${ }^{15}$ All interviews were transcribed verbatim and reviewed by the investigators (both medical experts and educated simulators). Subsequently, the interviews were analysed thematically by three qualitative researcher who created common themes across the transcripts. ${ }^{13}$ The generated main themes were reviewed and discussed among investigators in order enhance trustworthiness. The themes are presented in the result section.

\section{Patient and public involvement statement}

It was not appropriate or possible to involve patients or the public in the design, or conduct, or reporting, or dissemination plans of our research

\section{RESULTS}

In total 22 informants were included in this study (12 healthcare professionals, 7 medical experts,and 3 consultants responsible for the clusters). The informants perceived that the simulations resulted in positive experiences for the healthcare professionals and experienced the organisational changes as positive (ie, increase in interdisciplinary actions, decrease of bureaucracy, and a stronger sense of community). The following sections elaborate on these findings.

\section{Anxious concerns and demystification of the COVID-19}

The healthcare professionals in the interviews reported a feeling of uncertainty, partly due to being put in a stand-by position for emergency preparedness and partly due to the severity of the disease combined with an overwhelming amount of information. This is exemplified by a physiotherapist who participated in the simulation:

I feel like we have been caught in some kind of limbo or 'silence before the storm'... (Physiotherapist).

Here, in situ simulation was perceived as crucial in preparing for the COVID-19 disease by demystifying the disease and providing hands-on experiences with the patient category, effectively improving a sense of self-efficacy. ${ }^{16}$

Especially stress management was experienced as helpful in reducing potential stressors and increasing a sense of comfort in handling the COVID-19 patients, as also noted by the physiotherapist and a registered nurse:

...we were on such uncertain ground in the beginning. I also think [the simulation training] gave me some sense of security. (Physiotherapist)

Yes, in that sense it [COVID-19] was demystified. (Registered nurse)

The medical experts that facilitated the simulations highlighted the use of in situ simulations in order to enhance organisational learning and individual learning. Organisational changes, such as an increase in multidisciplinary cooperation and a stronger sense of community, prepared the clusters to the COVID-19 pandemic. Based on the simulations several changes were made, for example, how medical equipment was pre-packed in the COVID-19 clusters, how we organised the isolation rooms, the design of clinical teams as well as how to communicate in and out of quarantine areas. A medical expert pointed out such a specific change in organisation that was based directly on experience from the simulation:

During the simulations, we learned that it is a good idea to start each shift with a $1 \mathrm{~min}$ meeting in order 
to clarify roles if an acute situation in an isolation room should occur (Medical expert)

The medical experts also highlighted the need for individual learning of the healthcare professionals and perceived the simulation training as key in preparing oneself and the department for the COVID-19 pandemic:

In our cluster the simulations had a really good effect in order to demystify the disease and decrease fear among the healthcare professionals. The professionals had a lot of uncertainty in regards to what the pandemic would bring. Furthermore, the simulations raised a lot of questions that we, as heads of the cluster could answer in the daily meetings (Medical expert).

\section{Importance of multidisciplinary team training}

Another theme was the importance of interdisciplinary simulation sessions. The sessions highlighted each healthcare professional's value and role when handling COVID-19 patients. This was especially noted by the physiotherapist in the quote below, who experienced a stronger sense of professional identity, as well as an increased sense of comfort in teamwork:

[...] there has been a great experience of interdisciplinarity and an awesome feeling that we will handle it [COVID-19] together... (Physiotherapist).

In continuation, teamwork was enhanced during the simulations, according to a managing consultant:

The fact that experienced and un-experienced healthcare professionals were teamed up in the simulations really help in order to create insight into the value of each team-member. It also helped the new professionals to be integrated in departments, where they never had worked before. (Consultant responsible for department)

In continuation, consultants responsible for departments highlighted how the simulations helped ensuring a professional and calm working environment at the clusters. This is exemplified in the following quote by a consultant responsible of department who stressed how the simulations improved the working environment:

When we started the in situ simulation the atmosphere in the department became calmer. The treatment of COVID-19 patients is actually fairly simple, and the simulations helped the healthcare professionals to realize that. The simulations help them practice the COVID-19 treatment and makesome mistakes without jeopardizing patient-safety. It surely helps in order to calm the healthcare professionals and establish a very well-functioning department (Consultant responsible for department)

The positive gains of in situ simulation were also emphasised in the following quote by another head of department that did not receive any COVID-19 patients, but still valued the simulations.

This is surely something that we are going to use in the future (...) It has provided us with so much knowledge and teamwork. The content can be anything. It helped all of us because it is interdisciplinary and include experienced and unexperienced healthcare professionals. (Consultant responsible for department)

\section{Design and facilitation of the simulations}

The healthcare professionals highlighted the simulation facilitator as a key factor by being engaged in the scenario, ensuring fidelity and securing a safety net by debriefing the simulations. The medical experts that facilitated the simulations also highlighted the collaboration with in situ simulation experts in order to establish psychological safety ${ }^{17}$ and an optimal learning environment.

It makes it a lot easier for me as a facilitator, when there is an experienced in situ simulation instructor conducting the scenarios together with me. In this way, I know that the educational elements are being taken care of in a professional manner (Medical expert).

The presence of a medical expert and an educated simulation facilitator in each simulation secured the consistency among the facilitators. This is exemplified by one of the medical experts in the following quote:

The fact that we were two medical experts and one simulation expert to conduct all the in situ simulations in our department helped ensure consistency in the scenarios. A lot of the questions from the participants were the same and because we had daily meetings with the heads of the department, we knew what to answer. During the twoweeks we spend all our working time conducting these simulations, which made us confident in reaching the learning goals and establishing a smooth facilitation of the simulations. (Medical expert)

The informants in this study called for the establishment of a simulation task force across the hospital in order to share knowledge between departments and develop expertise in designing, implementing and facilitating the simulations.

I would have benefited by ending each day with an afternoon meeting with all the simulation facilitators from the COVID-clusters across the hospital. The pandemic caused a lot of questions in addition to facilitating the simulations. For example, what happens if a COVID-patient with comorbidity needs to be transferred to another department? If all simulation facilitators made a small daily report and shared this with the other facilitators, we could ensure knowledge sharing across the hospital. (Medical expert) 
Due to a lack of PPE, some simulations were conducted without the correct equipment in the initial phase of the simulation programme. During the simulation programme, the heads of departments realised the value of using the correct PPE, as explained in the quote below.

The outcome of simulation went from good to better, when we started to use the correct PPE, as this required that the healthcare team worked fully together. (Consultant responsible for department)

\section{DISCUSSION}

The interviews in this study suggest that in situ simulation enhanced teamwork, helped demystify the COVID-19 disease, and provided the healthcare professionals with competences within correct use of PPE and acute treatment of COVID-19 patients.

Healthcare professionals previously exhibit concern about family transmission of infectious diseases, thus, it seems reasonable if the COVID-19 pandemic had a negative impact on the healthcare professionals' perceived quality of work. ${ }^{18}$ Based on the findings in this study, it seems that in situ simulation can be a useful tool, when facing such a decrease in the perceived quality of work.

In medical education, Weller et al investigated effective healthcare teams and found an unacceptable rate of errors due to lack of teamwork between healthcare professionals. ${ }^{19}$ Consequently, they put forward seven interventions to overcome barriers to teamwork and team communication, including the use of simulation. Our study indicates that the informants experienced anxiety regarding the rapid spread of the COVID-19 virus. Furthermore, informants stated that in situ simulation made them feel more comfortable facing the task at hand that is, by demystifying the treatment of the COVID-19 disease and enhancing teamwork, all in a safe educational environment.

The uncertainty due to COVID-19 is likely to add complexity to the clinical work. Thus, training of healthcare professionals seems key in order to reduce stress and form coping strategies. Our findings align well with the stress model by Palmer et al, as their model highlight professionals' training in order to face an increased complexity of work and decrease stress. ${ }^{20}$

The hospital infrastructure seems influential when supporting the fundamental aim of well-being for all patients and delivering high standards of care. ${ }^{21}$ This is supported by our findings, suggesting that learning occurred on an organisation level as well as the individual level, as stated above. Systematic sharing of the insights, gained from the simulation, lead to changes in approaching COVID-19 patients in the isolation rooms, which reflects the organisational impact of the simulations. Similar findings are emphasised by Brydges et al, who advocate for the use of simulation when preparing for and responding to the early stages of the COVID-19 pandemic. $^{10}$
Simulation seems to have a potential in managing the global COVID-19 pandemic by rapidly facilitating hospital preparation and education of large numbers of healthcare professionals. ${ }^{7223}$ Wong et al advocated for the use of in situ simulation in the beginning of the pandemic in order to test the preparedness of isolation rooms, however, they do not specifically highlight using a coordinated and centralised simulation team to ensure the development of a robust curriculum development, as Dubé et al explicitly emphasise. ${ }^{624}$ While this study supports the use of simulation in a pandemic, the findings also reveal the need of coordinated planning across the hospital in order to secure that the learning goals of the simulation is reached. The coordination of simulation is highlighted by Brazil et al, who conducted an intervention similar to the one in this study, orchestrated by a simulation Service formally established across the hospital. ${ }^{25}$ The setting in this study would have benefited from the establishment of a simulation task force including educated simulating and medical experts, in order to ensure that the pedagogical, didactical and medical elements in the simulations where at the highest possible level. The main themes derived from the focus group interviews describe perception of in situ simulations from a variety of healthcare workers in an early stage of the pandemic. This timing seems important in understanding the need for simulation based educational activities prior to a healthcare crisis. However, due to the rapid development of the COVID-19 pandemic it was not possible to plan and organise the interviews in detail. Relatively few informants $(n=3$ in one group and $\mathrm{n}=4$ in another) reduce the generalisability of the results in this study. Furthermore, the circumstances did not allow us to conduct a study with objective assessment of skills learnt, for example, an examination or practical test of what was learned. Nonetheless, the in situ simulations are suggested to decrease stress and improve teamwork among the healthcare professionals. Similar, the simulations may have improved the clinical skills of the participating staff. It has not been possible to conduct a follow-up data collection in order to establish if demystification of the disease and decrease in stress still is present or new educational activities is needed.

\section{CONCLUSION}

In situ simulation may be useful to enhance learning on an organisation level as well as the individual level during a pandemic. This educational activity could serve an important role in facilitating hospital preparation and education of large numbers of healthcare professionals during a pandemic. The establishment of a simulation task force is, however, suggested as in situ simulation across a hospital requires coordination and rapid enrolment in healthcare crises.

\section{Author affiliations}

${ }^{1}$ Anaesthesiology, Aarhus Universitetshospital, Aarhus N, Denmark

${ }^{2}$ Dept of Medicine, Randers Regional Hospital, Randers NE, Denmark 
${ }^{3}$ Research Center for Emergency Medicine, Aarhus University Hospital, Aarhus N, Denmark

${ }^{4}$ Region Midtjylland Koncern HR Udvikling, Aarhus, Denmark

${ }^{5}$ Postgraduate medical education, Aarhus Universitetshospital, Aarhus, Denmark

${ }^{6}$ Department of Infectious Diseases, Aarhus Universitetshospital, Aarhus, Denmark

Acknowledgements The authors would like to express their gratitude to the 277 healthcare professionals who participated in the in situ simulations; the involved steering committees in the COVID-19 clusters for supporting the in situ simulations; Lone Dich and Thea Isaksen for transcription of data.

Contributors JJ and RDJ contributed with the idea of the study, collecting of data and the writing of the manuscript. BL, NT and LE contributed with collecting of data. GVE contributed with the idea of the study. All authors read and approved the manuscript before submission. The author acting as guarantor for the content of this study is JJ.

Funding The authors have not declared a specific grant for this research from any funding agency in the public, commercial or not-for-profit sectors.

Competing interests None declared.

Patient consent for publication Not applicable.

Ethics approval Participation in the focus group interviews was voluntary and participants' quotes were made anonymous. No ethical approval or trial registration was required for this study according to Danish legislation. All participants provided informed consent and gave permission to recording of the interviews.

Provenance and peer review Not commissioned; externally peer reviewed.

Data availability statement Data are available on reasonable request. Not applicable.

Supplemental material This content has been supplied by the author(s). It has not been vetted by BMJ Publishing Group Limited (BMJ) and may not have been peer-reviewed. Any opinions or recommendations discussed are solely those of the author(s) and are not endorsed by BMJ. BMJ disclaims all liability and responsibility arising from any reliance placed on the content. Where the content includes any translated material, BMJ does not warrant the accuracy and reliability of the translations (including but not limited to local regulations, clinical guidelines, terminology, drug names and drug dosages), and is not responsible for any error and/or omissions arising from translation and adaptation or otherwise.

Open access This is an open access article distributed in accordance with the Creative Commons Attribution Non Commercial (CC BY-NC 4.0) license, which permits others to distribute, remix, adapt, build upon this work non-commercially, and license their derivative works on different terms, provided the original work is properly cited, appropriate credit is given, any changes made indicated, and the use is non-commercial. See: http://creativecommons.org/licenses/by-nc/4.0/.

ORCID iD

Joachim Juelsgaard http://orcid.org/0000-0003-4389-3577

\section{REFERENCES}

1 World Health Organization [Internet], 2020. Available: https://www. who.int/data/stories/the-true-death-toll-of-covid-19-estimatingglobal-excess-mortality

2 Jensen RD, Bie M, Gundsø AP. Preparing an orthopedic department for COVID-19: lessons learned from reorganization and educational activities. Acta Orthop 2020:3674.
3 Vagni M, Maiorano T, Giostra V, et al. Coping with COVID-19: emergency stress, secondary trauma and self-efficacy in healthcare and emergency workers in Italy. Front Psychol 2020;11:566912.

4 Adams JG, Walls RM. Supporting the health care workforce during the COVID-19 global epidemic. JAMA 2020;323:1439-40.

5 Grasselli G, Pesenti A, Cecconi M. Critical care utilization for the COVID-19 outbreak in Lombardy, Italy: early experience and forecast during an emergency response. JAMA 2020;323:1545-6.

6 Dubé M, Kaba A, Cronin T, et al. COVID-19 pandemic preparation: using simulation for systems-based learning to prepare the largest healthcare workforce and system in Canada. Adv Simul 2020;5:1-12.

7 Dieckmann P, Torgeirsen K, Qvindesland SA, et al. The use of simulation to prepare and improve responses to infectious disease outbreaks like COVID-19: practical tips and resources from Norway, Denmark, and the UK. Adv Simul 2020;5:1-10.

8 Balikai SC, Badheka A, Casey A, et al. Simulation to train pediatric ICU teams in endotracheal intubation of patients with COVID-19. Pediatr Qual Saf 2021;6:e373.

9 Fregene TE, Nadarajah P, Buckley JF, et al. Use of in situ simulation to evaluate the operational readiness of a high-consequence infectious disease intensive care unit. Anaesthesia 2020;75:733-8.

10 Brydges R, Campbell DM, Beavers L, et al. Lessons learned in preparing for and responding to the early stages of the COVID-19 pandemic: one simulation's program experience adapting to the new normal. Adv Simul 2020;5:1-10.

11 Hunt EA, Duval-Arnould JM, Nelson-McMillan KL, et al. Pediatric resident resuscitation skills improve after "Rapid Cycle Deliberate Practice" training. Resuscitation 2014;85:945-51.

12 Kolbe M, Weiss M, Grote G, et al. TeamGAINS: a tool for structured debriefings for simulation-based team trainings. BMJ Qual Saf 2013;22:541-53.

13 Kvale S, Brinkmann S. Interviews: learning the craft of qualitative research interviewing. third. Los Angeles: SAGE Publications, 2015.

14 Stalmeijer RE, Mcnaughton N, Van Mook WNKA, . Using focus groups in medical education research: AMEE guide No. 91. Med Teach 2014;36:923-39.

15 Mattingly C, Dramas H, Plots $\mathrm{C}$. The narrative structure of experience. Cambridge: Cambridge Univesity Press, 1998.

16 Bandura A. Self-efficacy. New York: Academic Press, 1994: 4. 71-81.

17 Edmondson A. Psychological safety and learning behavior in work teams. Adm Sci Q 1999;44:350-83.

18 Walton M, Murray E, Christian MD. Mental health care for medical staff and affiliated healthcare workers during the COVID-19 pandemic. Eur Heart J Acute Cardiovasc Care 2020;9:241-7.

19 Weller J, Boyd M, Cumin D, Teams CD. Teams, tribes and patient safety: overcoming barriers to effective teamwork in healthcare. Postgrad Med J 2014;90:149-54.

20 Palmer S, Cooper C, Thomas K. Revised model of organisational stress for use within stress prevention/management and wellbeing programmes - Brief update. Int J Health Promot Educ 2003;41:57-8.

21 Luxon L. Infrastructure - the key to healthcare improvement. Future Hosp J 2015;2:4-7.

22 Brazil V. Translational simulation: not 'where?' but 'why?' A functional view of in situ simulation. Adv Simul 2017;2:1-5.

23 Lababidi HMS, Alzoraigi U, Almarshed AA. Simulation-Based training programme and preparedness testing for COVID-19 using system integration methodology. BMJ Simul Technol Enhanc Learn 2020.

24 Wong J, Goh QY, Tan Z, et al. Preparing for a COVID-19 pandemic: a review of operating room outbreak response measures in a large tertiary hospital in Singapore. Can J Anaesth 2020;67:732-45.

25 Brazil V, Lowe B, Ryan L, et al. Translational simulation for rapid transformation of health services, using the example of the COVID-19 pandemic preparation. Adv Simul 2020;5:1-10. 